Images du travail, travail des images

$9 \mid 2020$

L'apprentissage et l'enseignement professionnel en images

\title{
La lime et le chalumeau. Dans l'atelier de serrurerie de l'École Diderot à Paris en 1933
}

Guy Lambert

\section{OpenEdition}

\section{Journals}

Édition électronique

URL : http://journals.openedition.org/itti/536

DOI : 10.4000/itti.536

\section{Éditeur}

Université de Poitiers

\section{Référence électronique}

Guy Lambert, «La lime et le chalumeau. Dans l'atelier de serrurerie de l'École Diderot à Paris en 1933 », Images du travail, travail des images [En ligne], 9 | 2020, mis en ligne le 01 septembre 2020, consulté le 14 avril 2021. URL : http://journals.openedition.org/itti/536 ; DOI : https://doi.org/10.4000/itti.536

Ce document a été généré automatiquement le 14 avril 2021

Images du travail, travail des images 


\title{
La lime et le chalumeau. Dans l'atelier de serrurerie de l'École Diderot à Paris en 1933
}

\author{
Guy Lambert
}

À une date où l'iconographie de l'enseignement professionnel en appelle depuis longtemps au pouvoir évocateur des vues d'ateliers (Bodé, 2017), la place que celles-ci occupent dans la brochure de présentation de l'École Diderot publiée en 1933 témoigne de la confiance accordée à ces photographies pour incarner l'image de marque de l'établissement. À bien y regarder, si elles traduisent visuellement une aspiration à la modernité alors attendue d'un tel lieu d'enseignement, elles n'en illustrent pas moins l'ancienneté de cette institution scolaire, ouverte soixante ans plus tôt, première école d'apprentis de la Ville de Paris en 1873, qui peut à la fois se prévaloir de sa précocité, de son prestige et d'un bilan attesté par le nombre et la situation professionnelle de ses anciens élèves. Les clichés effectués pour cette publication par le studio Tourte et Petitin - spécialisé depuis 1882 dans la photographie scolaire de groupes d'élèves autant que d'édifices - livrent une vision avantageuse de l'école dont le rajeunissement $\mathrm{du}$ parc de machines et l'agrandissement des bâtiments reflètent les ambitions pédagogiques renouvelées (Legoux, 1972). Confronter les espaces ainsi montrés dans leur activité quotidienne conduit toutefois à relever dans ce discours visuel une part d'ambivalence, si ce n'est d'ambiguïté, dont témoigne tout particulièrement la vue de l'atelier de serrurerie. 
Image 1 : Atelier de serrurerie de l'École Diderot, en 1933

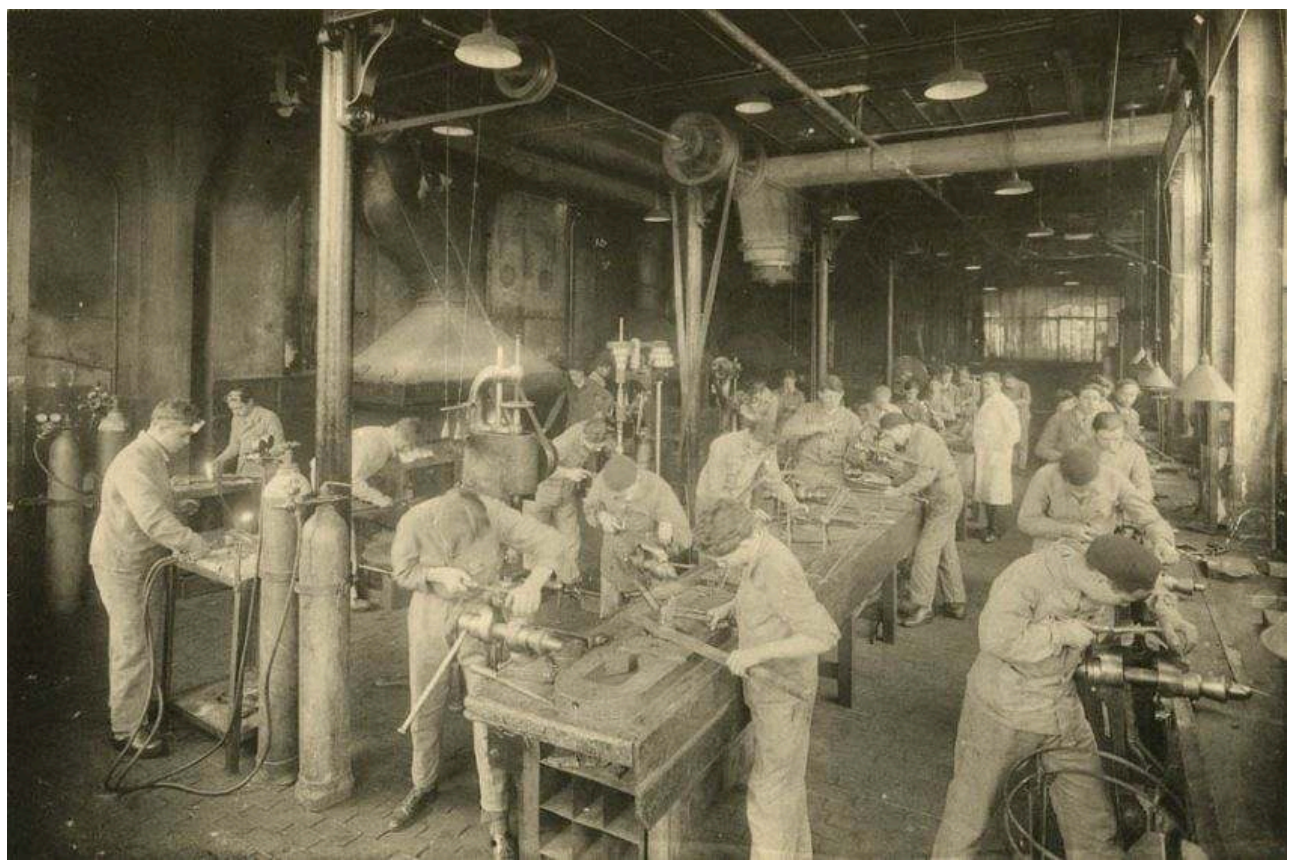

Extr. de École Diderot Paris 1933, Levallois, H. Tourte et M. Petitin, 1933.

Coll. part. Photographie Guy Lambert

2 Plus d'une vingtaine d'élèves s'affaire, chacun à son poste, ostensiblement absorbés par leur tâche pour ceux du premier plan, feignant d'ignorer l'opérateur auquel leurs camarades du fond - peut-être moins intimidés - sont plus tentés d'adresser un regard. La configuration des locaux et la volonté de les saisir dans leur ensemble dictent l'angle de la prise de vue, accentuant la perspective des établis implantés parallèlement aux fenêtres. Marquée par la file de colonnes en fonte, le sol pavé et le plancher en bois de l'étage supérieur, la physionomie des lieux tranche non seulement avec l'architecture des écoles nouvellement construites en France dans l'entre-deux-guerres, dont les photographies publiées dans la littérature spécialisée valorisent le caractère lumineux et dépouillé, mais également avec les corps de bâtiments les plus récents de l'École Diderot, recourant à la structure métallique et au béton armé. Conservant en grande partie les dispositions initiales de cette partie de l'établissement - installé lors de sa création sur le site d'une ancienne usine-, cet atelier témoigne d'une forme de stratification des réseaux de distribution d'énergie et de fluide, ajoutée parfois à celle des équipements propres aux métiers enseignés. Les arbres de transmission de la force qui traversent la pièce n'alimentant ici que quelques grands tours, les câbles et les conduites filant sous le plafond dessinent une trame moins dense que dans d'autres ateliers de l'école. En raison de l'importance de l'apprentissage manuel qui caractérise encore l'enseignement de la serrurerie tel qu'il est pratiqué dans ces murs, cet espace n'a pas connu de transformations aussi profondes que des sections comme celle des constructions électriques par exemple. 
Image 2 : Atelier de constructions électriques de l'École Diderot en 1933

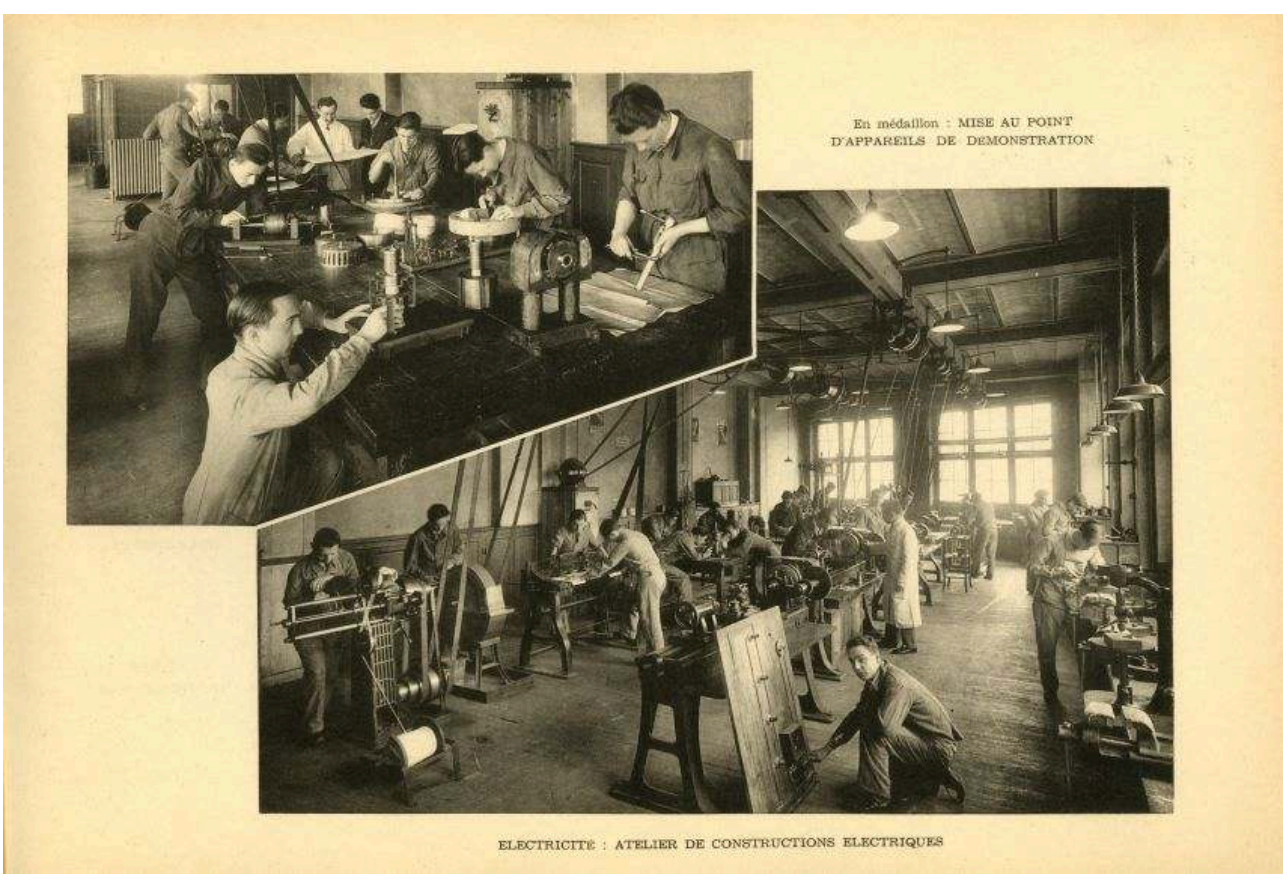

Extr. de École Diderot Paris 1933, Levallois, H. Tourte et M. Petitin, 1933.

Coll. part. Photographie Guy Lambert

3 Le maniement des outils par les élèves est indéniablement au cœur de la mise en scène, le geste prenant le pas sur le cadre, comme l'illustrent les coups de lime ou de scie bien assurés et les coups de marteau plus timides. L'échelonnement des tâches dans le champ de vision comme la gradation présumée de l'âge des élèves suggèrent un tableau idéalisé de la formation scolaire et de sa progression. Cette lecture semble confirmée par la succession des objets visibles sur l'établi, depuis les pièces simples coincées dans les étaux au premier plan - renvoyant aux exercices d'ajustage qui constituent les prémices de l'apprentissage - jusqu'aux travaux plus élaborés que l'œil devine plus loin, des guéridons en fer forgé en cours de fabrication, comme le révèle une vue de détail dans la brochure.

Peu visible sur cette photographie d'ensemble, hormis à travers les fers martelés sur le tas, le forgeage et son environnement sont renvoyés à la page suivante, montrant les deux foyers et les enclumes ici dissimulés au regard. Tranchant avec l'impression de faible renouvellement qui pourrait se dégager des lieux et des activités, la présence de postes de soudage autogène atteste l'attention portée par l'établissement aux renouvellements techniques et professionnels qui marquent alors le monde industriel. La position de cet outillage dans l'image, à la marge du cadrage comme à celle de l'atelier, ne reflète sans doute pas la place que sont appelés à prendre dans le cursus ces procédés d'assemblage métallique, qui font alors l'objet d'un nombre croissant d'expériences en France (Robert-Hauglustaine, 2000). L'installation dans cet atelier des bouteilles d'acétylène et d'oxygène ainsi que des chalumeaux renvoie peut-être autant à la mobilité de cet équipement qu'à la saturation dont souffrent les locaux de l'École Diderot. Si les élèves sont confrontés à plusieurs techniques de soudage, la soudure à l'arc est quant à elle hébergée par l'atelier de chaudronnerie, plus spacieux et d'aménagement plus récent, où elle trouve sans doute à s'intégrer aux exercices. Orienté vers la maitrise d'exécution des soudures, l'enseignement du soudage autogène 
s'accorde manifestement à la manière d'envisager la serrurerie. En définitive, jusque dans ses éléments les plus contemporains, l'image que renvoie cet atelier témoigne d'un attachement à l'apprentissage de l'habileté et de la précision, qui se rattache autant à l'idéal de formation à un métier complet qu'à un « dogme du travail manuel » (Legoux, op. cit., p. 237) portés par l'École Diderot depuis la fin du XIX ${ }^{\mathrm{e}}$ siècle et faisant sa réputation, même lorsque dans l'entre-deux-guerres la nécessité de former des techniciens pour l'industrie vient prendre le pas sur son ambition à produire des ouvriers d'élite.

\section{BIBLIOGRAPHIE}

Bodé G. (2017), « Les ateliers des écoles d'enseignement technique (années 1860-années 1950), un objet insaisissable? ", Histoire de l'éducation, n¹47, p. 37-65.

Legoux Y. (1972), Du Compagnon au technicien. L'École Diderot et l'évolution des qualifications, 1873-1972. Sociologie de l'enseignement technique français, Paris, Technique et vulgarisation.

Robert-Hauglustaine A.-C. (2000), « Les métiers du soudage en France et la création de filières de formation », Le Mouvement Social, n¹93, p. 43-59.

\section{AUTEUR}

\section{GUY LAMBERT}

Maître de conférences en Histoire et culture architecturales à l'École nationale supérieure d'architecture de Paris-Belleville (UMR AUSser). Ses recherches portent sur les interactions entre architecture, techniques et sociétés aux XIX ${ }^{\mathrm{e}}$ et XX $\mathrm{XX}^{\mathrm{e}}$ siècles, abordées sous l'angle de l'histoire culturelle et matérielle. En lien avec les thématiques de l'histoire de l'enseignement, il a notamment publié Une ambition pour les métiers. Paul Guadet \& André Boucton, l'architecture des écoles professionnelles (Morez, Musée de la Lunette, ENSAPB, 2013) et coordonné L'atelier et l'amphithéâtre. Les écoles de l'architecture, entre théorie et pratique (avec Estelle Thibault, Wavre, Mardaga, 2011) et Les lieux de l'enseignement technique. XIX'-XXe siècles (avec Stéphane Lembré, Histoire de l'éducation, $\mathrm{n}^{\circ}$ 147, 2017. 